\title{
5 Research Square

\section{P-selectin (CD62P) and soluble TREM-like transcript-1 (sTLT-1) are associated with coronary artery disease: A case control study}

Li Shen

Southern Medical University

Tianlun Yang

Central South University Xiangya School of Medicine

Ke Xia

Central South University Xiangya School of Medicine

Zhiqiang Yan ( $\square$ zqyanshanghai@gmail.com )

Southern Medical University fengxian hospital https://orcid.org/0000-0002-0184-2133

Juanjuan Tan

Shanghai Jiao Tong University School of Medicine

Lei Li

Southern Medical University

Yingchun Qin

shanghai university of traditional chinese medicine

\section{Research article}

Keywords: CAD, coronary artery disease; CD62P, platelet P-selectin ligand;sTLT-1, soluble TREM-like transcript-1; AMI, acute myocardial infarction, UAP, unstable angina pectoris; SAP, stable angina pectoris; ACS, acute coronary syndromes; NC,Normal control.

Posted Date: June 1st, 2020

DOI: https://doi.org/10.21203/rs.2.14618/v3

License: (c) (i) This work is licensed under a Creative Commons Attribution 4.0 International License. Read Full License

Version of Record: A version of this preprint was published on August 24th, 2020. See the published version at https://doi.org/10.1186/s12872-020-01663-2. 


\section{Abstract}

Background: Platelet activation plays a crucial role in the pathogenesis of coronary artery disease (CAD).Platelet P-selectin (CD62P) is a platelet classic activation indicator on the platelet surface,and soluble TREM-like transcript-1 (sTLT-1) is a new indicator,hpwever,_the relationship between these two markers and CAD(especially in acute coronary syndromes (ACS) has not been elucidated. This study aimed to investigate the expression of CD62Pon the platelet surface and STLT-1 in serum and to assess their relationship with CAD.

Methods: We measured the levels of CD62P and STLT-1 in 83 patients with CAD compared to 49 controls. The associations with age, blood pressure, lipid profiles, body mass index and liver injury marker levels were also examined.

Results: A stepwise increase in CD62P concentration was found based on the number of CAD patients ( $P$ $<0.01)$, especially in Acute myocaedial infarction $(A M I)$ patients $(P<0.01)$. Serum sTLT-1 concentration in the $\mathrm{AMI}$ and Unstable angina pectoris(UAP) groups was higher than that in the Normal control (NC) group $(P<0.01)$.

Conclusions: The consistency of sTLT-1 and CD62P expression levels in CAD indicates that the sTLT-1 level may be a new marker of platelet activation and is positively related to CAD.

\section{Introduction}

With an increasing global burden of coronary artery disease (CAD), early detection and timely management of risk factors are crucial to reduce the morbidity and mortality in these patients. Platelet activation plays a crucial role in the pathogenesis of $\mathrm{CAD}[1]$. Circulating-activated platelets are thought to trigger ischemic complications after angiography, angioplasty, and vascular surgery. The onset of CAD, especially in acute coronary syndromes (ACS), is closely associated with enhanced platelet adhesion and aggregation. Therefore, early detection and antiplatelet therapy are extremely important for the treatment of coronary heart disease.

P-selectin, also called CD62P, is a kind of glycoprotein stored in Weibel-Palade bodies of vascular endothelial cells and a-granules of platelets. Activated CD62P is a transmembrane protein expressed on the surface of platelets[2]. During platelet activation, the expression of CD62P on the surface of the cell membrane is dramatically increased, accompanied by a simultaneous increase in the expression level of plasma soluble CD62, which plays an important role in the initiation, formation, and expansion of the thrombus. P-selectin (CD62p) exposure is an established marker for platelet activation.Current research has found that percentage expression of P-selectin on platelets without any stimulation with platelet activating factors (ADP, collagen and thrombin) was higher in CAD patients compared to controls[3]. Tenaglia et al.[4] reported significantly greater P-selectin staining on atherectomy specimens from patients with unstable angina than from patients with stable angina.These findings support the concept 
that the increased expression of P-selectin is important in atherosclerosis and targeting this pathway may be a therapeutic option.

TREM-like transcript-1 (TLT-1), coexistence of CD62P in platelet alpha particles, is a new platelet activation indicator expression in the platelet membrane surface[5,6]at the same time, releasing a soluble TLT-1 (sTLT-1) in the plasma, enhances the aggregation of platelet[2,6], which can also reflect the expression of platelet membrane TLT-1[7]. Despite the proof of the biological function of CD62P in platelets and STLT-1 in serum, there is little information about their role in patients with CAD. Therefore, this study was performed to elucidate the association between CD62P, sTLT-1, and CAD. Based on the potential link between CD62P, sTLT-1, and platelet activation, we systematically analyzed their correlation with CAD.

\section{Methods}

\section{Clinical samples}

This study enrolled consecutive patients with CAD who had Coronary angiographic stenosis was greater than $50 \% \varangle 83 \rrbracket$. Among them, 29 had acute myocardial infarction (AMI), 26 had unstable angina pectoris (UAP), and 28 had stable angina pectoris (SAP). Patients were conpared with 49 healthy control subjects from the physical examination center without CAD. Patients with any evidence of tumors or systemic disease were excluded, The patients agreed to participate in this study and signed the informed consent form. A detailed clinical history was collected from all of the patients, including clinical characteristics.

\section{Information collection}

Clinical data of the general condition (age, gender, height, and weight), medical history (hypertension, diabetes, or stroke), personal history (smoking or drinking), medication history, family history, and laboratory tests were obtained from all patients.

\section{Measurement of plasma}

Blood venous samples were collected directly in plastic tubes in the morning. The collected blood samples were about $5 \mathrm{ml}$ and they placed in a vacuum blood collection vessel, centrifuged for $3 \mathrm{~min}(1500 \mathrm{r} / \mathrm{min})$ to separate the upper platelet-rich plasma, and placed in a sterile Epoxy epoxide(EP) tube. The expression level of CD62P on the platelet surface was detected by flow cytometry (BD FACSCalibur, America). The concentration of sTLT-1 in serum was measured by the enzyme-linked immunosorbent assay according to the manufacturer's instructions.

\section{Statistical analysis}


The statistical analysis was performed by IBM SPSS version 22.0 software. Quantitative data are presented as mean $\pm S D$ deviation. Any differences among $\geq 3$ groups were evaluated by ANOVA with Scheffe's test for parametric variables and by the Kruskal-Wallis test with the Steel-Dwass test for nonparametric variables. Any differences between 2 groups were evaluated by the unpaired $t$ test for parametric variables. Correlations between STLT-1, CD62P, and the severity of coronary atherosclerosis were evaluated by Spearman's rank correlation test. A P-value level of $<0.05$ was considered to indicate statistically significance.

\section{Result}

\subsection{Clinical characteristics of the participants}

The details of demographic, clinical, and laboratory data of the 132 study participants are listed in Table 1. The results showed that there were no significant differences in age, sex, body mass index ( $\mathrm{BMI})$, systolic blood pressure,diastolic blood pressure( DBP ), and platelet count between the CAD and NC groups.Low density lipoprotein( LDL ) and triglycerides ( TG )levels in the ACS group were higher than those in the NC group $(\triangle P<0.05)$. A stepwise decrease in high density lipoprotein ( $H D L)$ levels was found in the AMI and UAP groups than in the control group $(\triangle P<0.05)$. The blood sugar(BS )level in the AMI

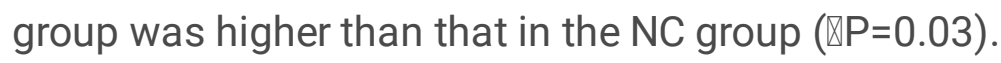

2. 2 Levels of plasma sTLT-1 and platelet CD62P in patients with CAD and healthy controls.

We found that the expression level of CD62P in the platelet membrane in the CAD group was significantly higher than that in the NC group, and the difference was statistically significant (Figure1a-e and Table 1). The expression level of platelet CD62P in the AMI group was significantly higher than that in the SAP group $(P=0.011)$, and the difference was statistically significant $(P<0.01)$.

Given that elevated plasma levels of STLT-1 are implicated in the activation of platelets, we measured theplasma sTLT-1 levels in patients diagnosed with CAD. Soluble TLT-1 levels were significantly increased in patients with AMI and UAP compared with matched,healthy control participants; a significant correlation was observed between patients with AMI versus those with $\operatorname{SAP}(P<0.01)$.

2.3 Correlation analysis of serum sTLT, platelet membrane CD62P, and other risk factors of coronary heart disease

Spearman regression was used to analyze the correlation between platelet membrane CD62P and serum STLT-1 and the other risk factors of coronary heart disease in ACS patients, including HDL, LDL, and TG (Tables 3 and 4). The results showed that the serum sTLT-1 level was positively correlated with TG $(r=0.42), L D L(r=0.17)$, and CD62P ( $r=0.49)$, and it was negatively correlated with HDL $(r=-0.28)$. Among thest factors, there was a positive correlation of sTLT-1 with BS ( $r=0.2), \mathrm{CHO}(r=0.47), \mathrm{LDL}(r=0.32)$, body weight $(r=0.21)$, and sTLT-1 $(r=0.49)$, but there was a negative correlation of sTLT-1 with HDL $(r=-0.29)$. 


\section{Discussion}

The World Health Organization (WHO) divides coronary heart disease into the following five clinical types: asymptomatic, angina pectoris, myocardial infarction, ischemic cardiomyopathy, and sudden death. ACS is referred to as coronary atherosclerosis plaque rupture and thrombosis, or vasospasm and clinical syndrome. Acute or subacute myocardial ischemia includes UAP and AMI. The typical presentation of myocardial ischemia is chest pain, and its development is associated with platelet activation. The adhesion molecule CD62P plays a dominant role in modulating the interactions between platelets and the endothelium, and it is involved in acute cardiovascular events[8]. Studies have confirmed that CD62P is one of the classic indicators of platelet activation. The activation of platelets is one of the targets of antiplatelet therapy.

The results of our study showed that the platelet CD62P level in the AMI, UAP, and SAP groups was significantly higher than that in the normal control group, and the platelet CD62P level in the AMI group was significantly higher than that in the UAP and SAP groups, which was consistent with previous research results. The results of this experiment showed that there was no significant difference between the UAP group and the SAP group, which may be related to the small number of specimens collected in this experiment.

TLT- 1 is a type- 1 transmembrane protein $[9,10,6]$, which is highly and exclusively expressed in megakaryocytes and platelets[11], stored in a-granules and possibly in another platelet compartment, and is rapidly upregulated on the surface of activated platelets, with CD62P showing the most obvious increase in expression [12]. Upon platelet activation, TLT-1 transports to the membrane where it enhances $\mathrm{Ca} 2+$ influx and promotes platelet aggregation. TLT-1 is a new player in platelet aggregation.It has been proved that the expression level of STLT-1 in patients with chest pain is significantly higher than that in the control group[13,14]. In acute respiratory distress syndrome, the results showed that the expression level was closely related to coronary heart disease, and it was also one of the indexes of platelet activation. In a murine model of acute lung injury (ALI), Morales-Ort' Iz et al. showed that TLT-1 mediates fibrinogen deposition in the lungs and facilitates platelet-neutrophil release during transmigration[15]. In mice, inhibition of TLT-1 has been shown to reduce thrombosis in carotid artery thrombosis models and to protect mice from excessive bleeding during pulmonary embolism[13]. It has been proved that the serum level of sTLT-1 is correlated with the incidence of ACS and has a certain predictive value[16]. The specific mechanism needs to be studied further. Combined with our experimental results, the serum sTLT1 level in the AMI group and the UAP group was significantly higher than that in the NC group and the SAP group. The expression level of sTLT-1 in the AMI group was significantly higher than that in the SAP group. Therefore, it is speculated that in the early stage of rapid platelet activation, TLT-1 may also show an obvious increase in expression as CD62P, which is one of the indicators of platelet activation. The level of serum STLT-1 in ACS patients can provide some reference for the diagnosis of coronary heart disease. Antiplatelet therapy is the cornerstone of the treatment for thrombotic diseases, especially ACS. This study undoubtedly explores the platelet function in CAD. TLT-1 is a newly discovered indicator of 
platelet activation, and further study and better understanding of this indicator may provide new directions and targets for the diagnosis of thrombosis-related diseases and anti-platelet therapy.

\section{Conclusion}

The consistency of sTLT-1 and CD62P expression levels in CAD indicates that the sTLT-1 level may be a new marker of platelet activation and is positively related to CAD, especially in ACS patients. The serum TLT-1 level in patients with coronary artery disease (especially ACS) syndrome was significantly higher than that in the normal population, suggesting that TLT-1 may be involved in the disease occurrence in patients with ACS.

\section{Declarations}

\section{Ethics approval and consent to participate}

Participants gave written informed consent before the study, this study was approved by the Medical Ethics Committee of Xiangya Hospital of Central South University (approval NO: 201192) and in accordance with the Declaration of Helsinki.

\section{Availability of data and materials}

The datasets used and/or analysed during the current study are available from the corresponding author on reasonable request.

\section{Consent for publication}

All the participants provided written informed consent for the publication of the results of this study.

\section{Competing interests}

The authors declare that they have no complict interests.

\section{Authors' contributions}

$S L$ interpreted the data and wrote the manuscript. Y ZQ and $Y$ TL designed the study protocol and supervised the project. L L measured the serum sTLT-1 levels using ELISA kits and CD62P levels using Flow cytometry. Q YC and T JJ enrolled and managed the participants. X K collected the data and edited the article. All of the authors read and approved the final manuscript.

\section{Acknowledgments}

We thank all of the study participants. We also appreciate the contributions of the nurses and doctors at the Department of Cardiology,Xiangya Hospital,Central south University in this study. 


\section{Funding}

This work were supported by the grant from National Nature Science Foundation of china,grant number(31570949).

\section{References}

1 Grande P, Grauholt AM, Madsen JK. Unstable angina pectoris. Platelet behavior and prognosis in progressive angina and intermediate coronary syndrome. CIRCULATION. 1990;81(1 SuppI):I16-9, I22-3

2Frenette PS, Denis CV, Weiss L, Jurk K, Subbarao S, Kehrel B et al. P-Selectin glycoprotein ligand 1 (PSGL-1) is expressed on platelets and can mediate platelet-endothelial interactions in vivo. J EXP MED. 2000;191(8):1413-22. 'doi:'10.1084/jem.191.8.1413.

3George R, Bhatt A, Narayani J, Thulaseedharan JV, Sivadasanpillai H, Tharakan JA. Enhanced P-selectin expression on platelet-a marker of platelet activation, in young patients with angiographically proven coronary artery disease. MOL CELL BIOCHEM. 2016;419(1-2):125-33. 'doi:'10.1007/s11010-016-2756-4.

4Tenaglia AN, Buda AJ, Wilkins RG, Barron MK, Jeffords PR, Vo K et al. Levels of expression of P-selectin, E-selectin, and intercellular adhesion molecule-1 in coronary atherectomy specimens from patients with stable and unstable angina pectoris. AM J CARDIOL. 1997;79(6):742-7. 'doi:'10.1016/s00029149(96)00861-2.

5Washington AV, Schubert RL, Quigley L, Disipio T, Feltz R, Cho EH et al. A TREM family member, TLT-1, is found exclusively in the alpha-granules of megakaryocytes and platelets. BLOOD. 2004;104(4):1042-7. 'doi:'10.1182/blood-2004-01-0315.

6Washington AV, Quigley L, McVicar DW. Initial characterization of TREM-like transcript (TLT)-1: a putative inhibitory receptor within the TREM cluster. BLOOD. 2002;100(10):3822-4. 'doi:'10.1182/blood2002-02-0523.

7Derive M, Bouazza Y, Sennoun N, Marchionni S, Quigley L, Washington V et al. Soluble TREM-like transcript-1 regulates leukocyte activation and controls microbial sepsis. J IMMUNOL. 2012;188(11):5585-92. 'doi:'10.4049/jimmunol.1102674.

8Koh TW, DeSouza AC, Pepper JR. The adhesion molecule P-selectin and cardiovascular diseasecardiac surgical implications. EUR HEART J. 2004;25(11):993. 'doi:'10.1016/j.ehj.2004.01.023.

9Cohen J. The immunopathogenesis of sepsis. NATURE. 2002;420(6917):885-91.

'doi:'10.1038/nature01326.

10Washington AV, Schubert RL, Quigley L, Disipio T, Feltz R, Cho EH et al. A TREM family member, TLT-1, is found exclusively in the alpha-granules of megakaryocytes and platelets. BLOOD. 2004;104(4):1042-7. 'doi:'10.1182/blood-2004-01-0315. 
11 Jolly L, Lemarie J, Carrasco K, Popovic B, Derive M, Boufenzer A et al. Triggering Receptor Expressed on Myeloid cells-1: a new player in platelet aggregation. Thromb Haemost. 2017;117(9):1772-81. 'doi:'10.1160/TH17-03-0156.

12 Geng JG, Chen M, Chou KC. P-selectin cell adhesion molecule in inflammation, thrombosis, cancer growth and metastasis. CURR MED CHEM. 2004;11(16):2153-60. 'doi:'10.2174/0929867043364720.

13Esponda OL, Hunter R, Del RJ, Washington AV. Levels of soluble TREM-like transcript 1 in patients presenting to the emergency department with chest pain. Clin Appl Thromb Hemost. 2015;21(1):30-4. 'doi:'10.1177/1076029614547298.

14Morales-Ortiz J, Rondina MT, Brown SM, Grissom C, Washington AV. High Levels of Soluble Triggering Receptor Expressed on Myeloid Cells-Like Transcript (TLT)-1 Are Associated With Acute Respiratory Distress Syndrome. Clin Appl Thromb Hemost. 2018;24(7):1122-7. 'doi:'10.1177/1076029618774149.

15Senis YA. TLT-1: please release me, let me go. BLOOD. 2018;132(23):2427-9. 'doi:'10.1182/blood-201810-879676.

16Fu R, Song X, Su D, Li S, Gao L, Ji C. Serum STLT-1 and bilirubin levels in patients with acute coronary syndrome and correlation with prognosis. EXP THER MED. 2018;16(1):241-5. 'doi:'10.3892/etm.2018.6137.

\section{Tables}

Table 1. Information on clinical characteristics (Mean \pm SD) 


\begin{tabular}{lllll}
\hline & & SAP(n=28) & & AMI (n=26) \\
& NC (n=49) & & UAP (n=29) & \\
\hline Age (years) & $55.10 \pm 9.19$ & $56.89 \pm 8.59$ & $57.28 \pm 8.69$ & $58.62 \pm 7.52$ \\
/Men/female (n) & $(27 / 22)$ & $(15 / 13)$ & $(16 / 13)$ & $(15 / 11)$ \\
\hline BMI (KG/m2) & $23.24 \pm 3.06$ & $24.11 \pm 1.28$ & $24.33 \pm 1.96$ & $24.19 \pm 2.43$ \\
\hline TG (mmol/L) & $1.12 \pm 0.53$ & $1.21 \pm 0.59$ & $1.52 \pm 0.76_{\triangle}$ & $1.76 \pm 1.06_{\triangle}$ \\
\hline LDL (mmol/L) & $2.24 \pm 0.55$ & $2.38 \pm 0.65$ & $2.81 \pm 0.43_{\triangle 0}$ & $2.99 \pm 0.43_{\triangle 0}$ \\
\hline HDL (mmol/L) & $1.43 \pm 0.44$ & $1.32 \pm 0.32$ & $1.14 \pm 0.23_{\triangle 0}$ & $1.10 \pm 0.26_{\triangle 0}$ \\
\hline BS (mmol/L) & $5.04 \pm 0.53$ & $5.3 \pm 0.53$ & $5.37 \pm 0.96$ & $5.72 \pm 1.52$ \\
\hline ALT (IU/L) & $28.58 \pm 7.01$ & $26.08 \pm 6.05$ & $40.23 \pm 15.38$ & $43.12 \pm 19.83$ \\
\hline SBP (mmHg) & $125.8 \pm 20.02$ & $130.5 \pm 19.24$ & $131.4 \pm 22.35$ & $129.3 \pm 19.03$ \\
\hline DBP (mmHg) & $79.08 \pm 8.05$ & $80.32 \pm 8.59$ & $76.65 \pm 11.28$ & $77.38 \pm 12.66$ \\
\hline Platelet count (109/L) & $193.1 \pm 44.47$ & $191.2 \pm 46.55$ & $185.93 \pm 45.24$ & $187.12 \pm 42.48$ \\
\hline
\end{tabular}

Abbreviations: BMI: body mass index; TG: total triglycerides; LDL-C: lowdensity lipoprotein cholesterol; HDL-C: high-density lipoprotein cholesterol; Glu: fasting glucose; BS: blood glucose; ALT: alanine aminotransferase; SBP: systolic blood pressure; DBP: diastolic blood pressure; Data are presented as mean values \pm standard or proportions (\%). P-value represents the difference among the four groups: vs NC: ${ }_{\triangle} \mathrm{P}<0.05$; vs SAP: ${ }^{\mathrm{P}}<0.05$; vs $\mathrm{NC}:{ }_{ } \mathrm{P}=0.03$;

Table 2. Levels of plasma sTLT-1 and platelet CD62P in patients with CAD and healthy controls

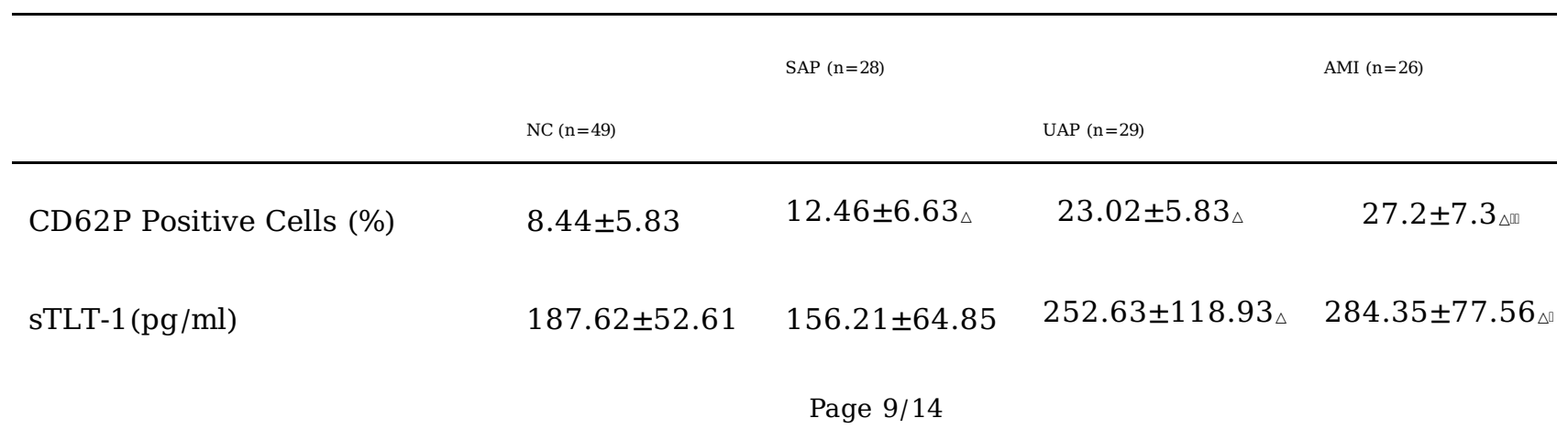


Abbreviations: CD62P: platelet P-selectin; sTLT-1: soluble TREM-like transcript-1; Data are presented as mean values \pm standard or proportions (\%). P-value represents the difference among the four groups: vs NC: ${ }_{\triangle} \mathrm{P}<0.05$; vs UAP : ${ }_{\mathbb{P}} \mathrm{P}=0.011$; vs $\mathrm{SAP}:{ }_{\mathbb{}} \mathrm{P}<0.01$

1

Table 3. Correlation analysis of serum sTLT and other risk factors of CAD 
STLT-1

\begin{tabular}{ccc}
\cline { 2 - 3 } & $\mathrm{r}$ & P-value \\
\hline BS & 0.18 & 0.07 \\
\hline LDL & 0.42 & 0.00 \\
\hline HDL & 0.17 & 0.05 \\
\hline Pt & -0.28 & 0.00 \\
\hline Age & -0.077 & 0.34 \\
\hline Weight & 0.15 & 0.09 \\
\hline BMI & 0.26 & 0.77 \\
\hline Sex & 0.33 & 0.71 \\
\hline CD62P & -0.15 & 0.14 \\
\hline S & 0.49 & 0.00
\end{tabular}

r: Spearman's correlation

coefficients

Table 3. Correlation analysis of The platelet membrane CD62P and other risk factors of

CAD

\begin{tabular}{|c|c|c|}
\hline & \multicolumn{2}{|c|}{ CD62P } \\
\hline & $\mathrm{r}$ & P-value \\
\hline BS & 0.25 & 0.04 \\
\hline TG & 0.38 & 0.65 \\
\hline LDL & 0.32 & 0.00 \\
\hline HDL & -0.29 & 0.00 \\
\hline $\mathrm{Pt}$ & -0.27 & 0.61 \\
\hline Age & 0.13 & 0.15 \\
\hline Weight & 0.21 & 0.02 \\
\hline BMI & 0.29 & 0.04 \\
\hline Sex & 0.12 & 0.22 \\
\hline
\end{tabular}


r: Spearman's correlation coefficients

\section{Figures}
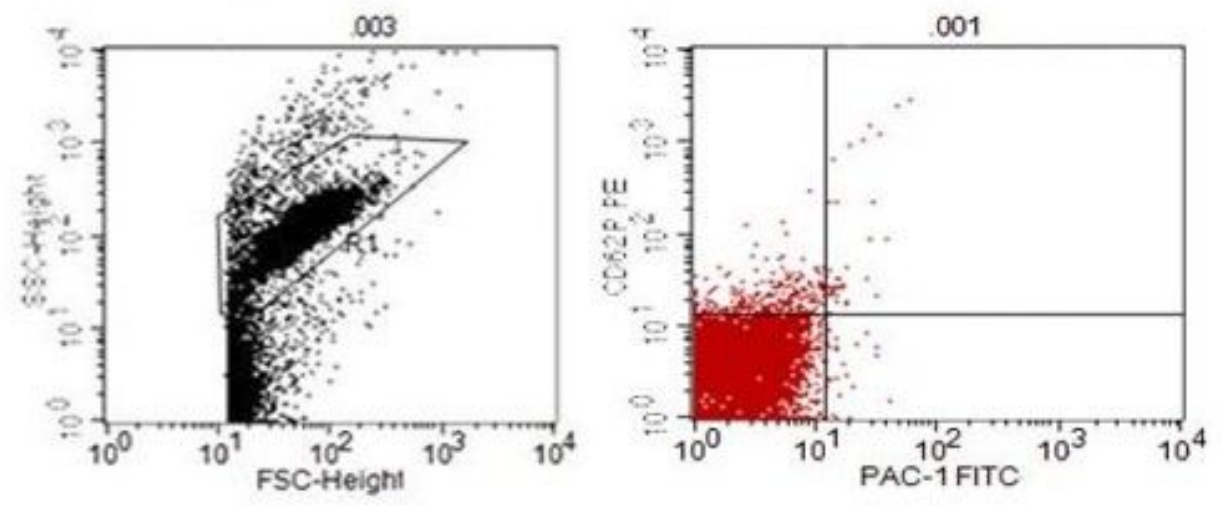

a.Platelet particles were selected on the FSC histogram

b. Positive rate of platelet CD62P in NC group (\%)
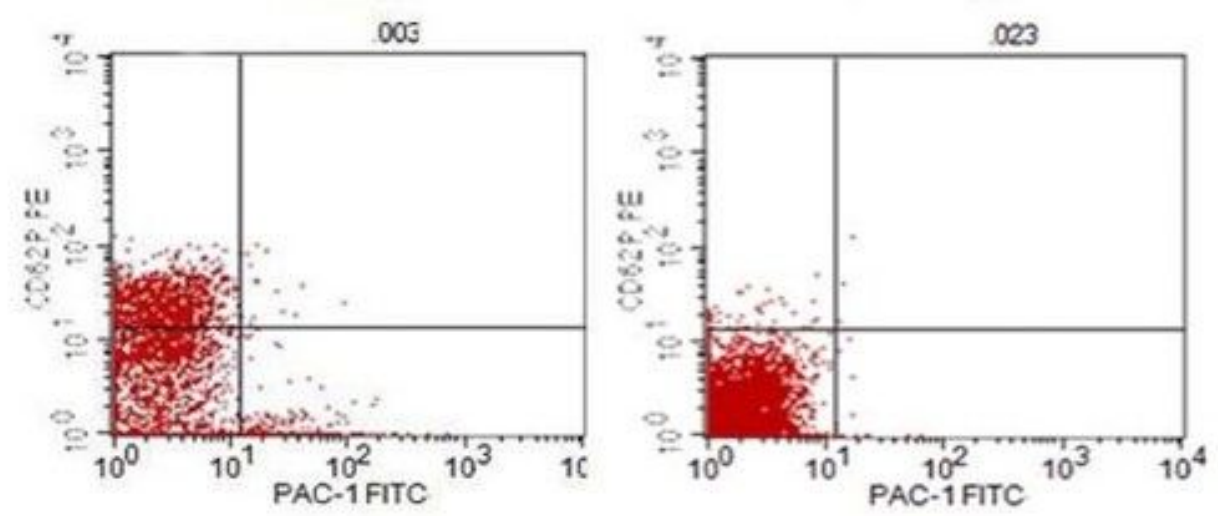

C.Positive rate of platelet CD62P in SAP group (\%)

D. Positive rate of platelet CD62P in

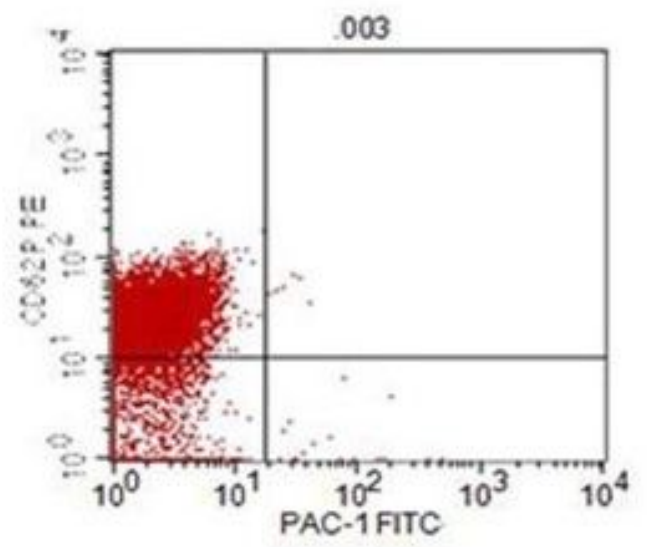

E Positive rate of platelet CD62P in AMI group (\%) 


\section{Figure 1}

A region (R1) is drawn around the platelets, which were identified according to their characteristic size (a); The CD62P positivity rate of platelets in the NC, SAP, UAP, and AMI groups (\%) (b-e).

\section{$P<0.001$}

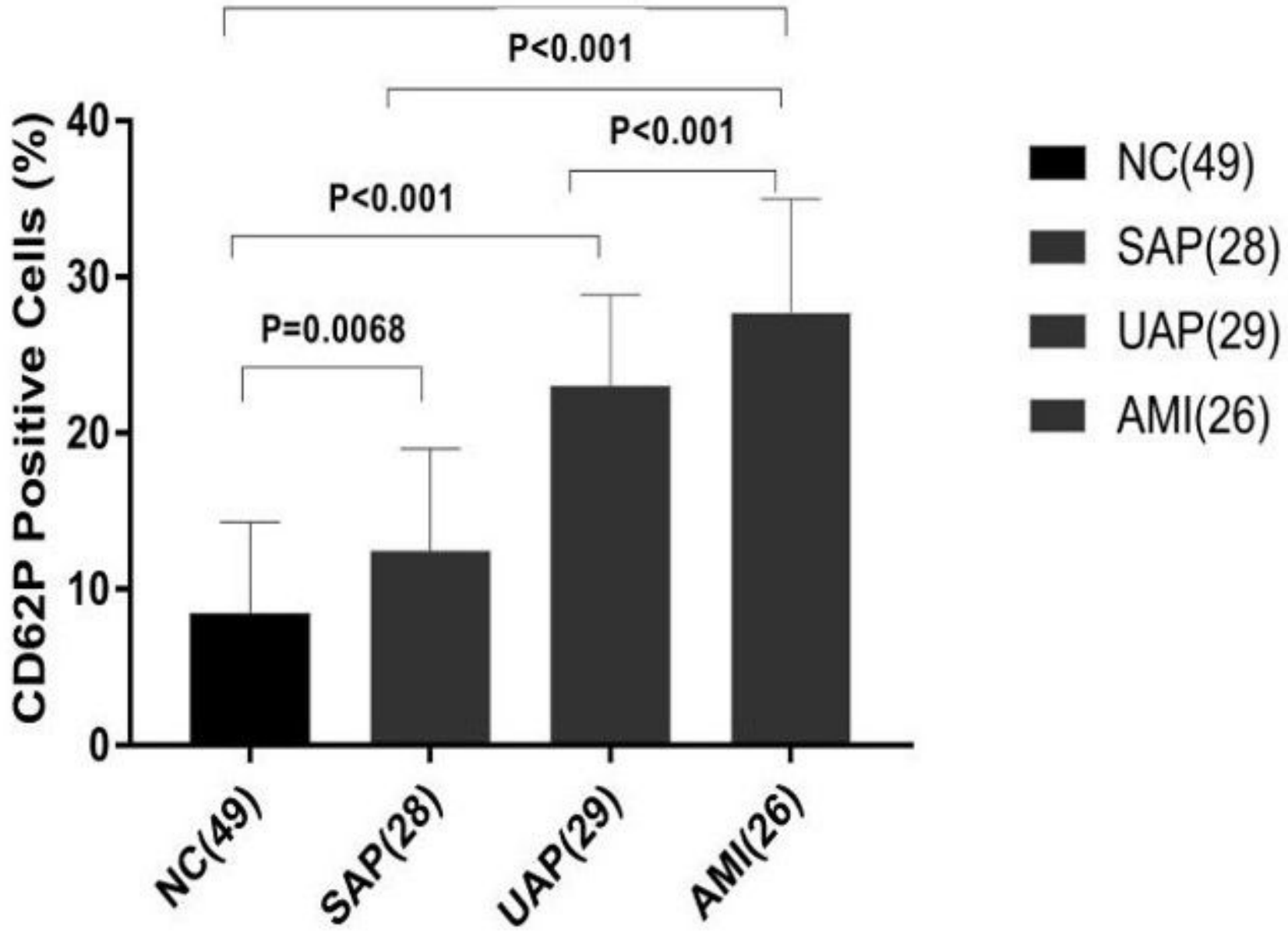

Figure 2

Comparison of the positivity rate of CD62P in the platelet membrane in each group (NC group, SAP group, UAP group, and AMI group): The CD62P levels were significantly higher in CAD participants: NC vs SAP: $P=0.0068$; vs UAP: $P<0.001$; vs AMI: $P<0.001$; especially in AMl: vs SAP: $P<0.001$; vs UAP: $P<0.001$. 


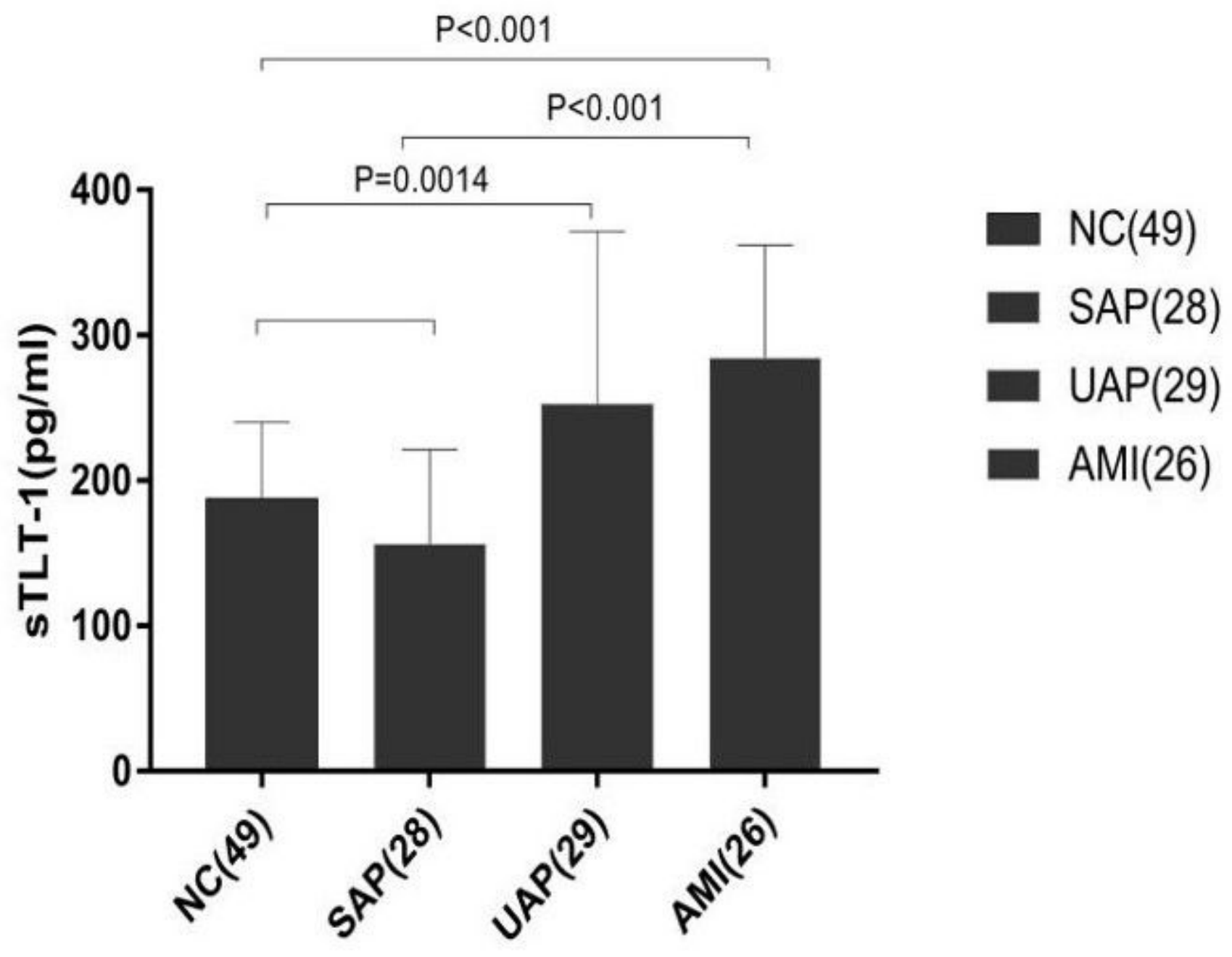

Figure 3

Serum sTLT-1 level was compared among the four groups (NC group, SAP group, UAP group, and AMI group): The sTLT-1 levels weres ignificantly higher in CAD participants: NC vs SAP: $P=0.0235$; vs UAP: $p=0.0014$; vs AMI: $P<0.001$; especially in AMl: vs: SAP: $P<0.001$.

\section{Supplementary Files}

This is a list of supplementary files associated with this preprint. Click to download.

- renamedbf871.xls

- renamede52e4.xls 